\title{
An overview of the effect of sodium glucose cotransporter 2 inhibitor monotherapy on glycemic and other clinical laboratory parameters in type 2 diabetes patients
}

\author{
This article was published in the following Dove Press journal: \\ Therapeutics and Clinical Risk Management \\ 15 July 2016 \\ Number of times this article has been viewed
}

\author{
Yaowen Wang' \\ Xueting $\mathrm{Hu}^{2}$ \\ Xueying $\mathrm{Liu}^{3}$ \\ Zengqi Wang ${ }^{2}$ \\ 'Department of Clinical Laboratory, \\ Weifang People's Hospital, \\ ${ }^{2}$ Department of Clinical Laboratory, \\ Weifang Traditional Chinese Hospital, \\ Weifang, ${ }^{3}$ Department of Clinical \\ Laboratory, The Third Hospital of \\ Jinan, Jinan, People's Republic of China
}

Correspondence: Zengqi Wang Department of Clinical Laboratory, Weifang Traditional Chinese Hospital, I055 Weizhou Road, Weifang 26I04I, People's Republic of China

Tel +86 I58 64599659

Email wangzengqiop@sohu.com
Objectives: We aimed to determine the effect of sodium glucose cotransporter 2 (SGLT2) inhibitor monotherapy on glycemic and other clinical laboratory parameters versus other antidiabetic medications or placebo therapy in patients with type 2 diabetes mellitus. In addition, we aimed to investigate the risk of diabetic ketoacidosis associated with SGLT2 inhibitor therapy and evaluate its weight-sparing ability.

Design: Meta-analysis.

Materials and methods: PubMed and MEDLINE were searched to identify eligible studies up to December 2015. Randomized controlled trials that assessed the efficacy and safety of SGLT2 inhibitor monotherapy versus placebo therapy or active control were considered. The Cochrane Collaboration Risk of Bias Tool was used to evaluate quality and bias. The mean difference was used to evaluate the glycemic and other clinical laboratory parameters for SGLT2 inhibitor intervention versus control by drugs or placebo. Similarly, the risk ratio was used to assess adverse events, and the $I^{2}$ was used to evaluate heterogeneity.

Results: SGLT2 inhibitors significantly decreased glycated hemoglobin (HbA1c) $(P<0.001)$, weight $(P<0.001)$, and the low-density lipoprotein/high-density lipoprotein ratio $(P=0.03)$ compared with placebo therapy. No statistically significant changes were found in fasting plasma glucose, 2-hour postprandial glucose, or lipid parameters. Significant changes in the uric acid level were found for SGLT2 inhibitors versus placebo therapy $(P=0.005)$ or active control $(P<0.001)$. Although no significant change in levels of ketones occurred $(P=0.93)$, patients receiving SGLT2 inhibitors were at greater risk of increased ketone bodies. Events suggestive of urinary tract infection and pollakiuria presented the greatest risk for patients receiving SGLT2 inhibitors versus active control or placebo therapy.

Conclusion: SGLT2 inhibitors significantly decreased HbA1c, body weight, and the lowdensity lipoprotein/high-density lipoprotein ratio and were found to be safe and well tolerated in type 2 diabetes mellitus patients. Further randomized control trials are required to establish their risk for ketoacidosis.

Keywords: SGLT2 inhibitor, diabetic ketoacidosis, type 2 diabetes mellitus, hyperglycemia, dyslipidemia, weight loss

\section{Introduction}

The worldwide prevalence of type 2 diabetes mellitus (T2DM) is expected to increase by $89 \%$ in the next 2 decades, and the role of the renal system in this context has been studied only recently. ${ }^{1,2}$ In the kidneys, the glomeruli filter $\sim 180 \mathrm{~g}$ of glucose from 
the blood per day; however, renal tubular epithelial cells reabsorb most of this glucose from the proximal tubule, thus maintaining homeostasis of glucose. ${ }^{3}$ In diabetic patients, the kidneys increase the maximum transport rate for glucose, thus decreasing glycosuria and exacerbating hyperglycemia. ${ }^{3}$

Sodium glucose cotransporters, which belong to a family of adenosine triphosphate-dependent proteins, are located in the S1, S2, and S3 segments of the proximal tubule and mediate the resorption of glucose. ${ }^{3}$ In particular, sodium glucose cotransporter 2 (SGLT2), a low-affinity, high-capacity transporter in the S1 segment, is responsible for resorbing $90 \%$ of the filtered glucose. ${ }^{3}$ Therefore, some patients with mutations in the SGLT2 gene experience increase or decrease in glycosuria that contributes to hypo- or hyperglycemia. ${ }^{3}$ Increased genetic expression and activity of SGLT2 are associated with an increase in the maximum resorption capacity (and the maximum transport rate) of glucose during the hyperglycemic episodes that occur in patients with T2DM.,4

Orally administered SGLT2 inhibitors are a novel class of antidiabetic agents designed to address the unmet needs of patients with T2DM. Human trials have shown that these inhibitors increase control of glucose by decreasing levels of blood glucose and glycated hemoglobin (HbA1c), irrespective of the insulin levels or sensitivity., 4 Therefore, they can be combined with all other classes of antidiabetic medications, including exogenous insulin. ${ }^{4,5}$ Side effects, such as weight gain and hypoglycemia, often occur with traditional treatments for T2DM and may negate the benefits of decrease in blood glucose offered by these treatments. ${ }^{5}$ However, the SGLT2 gene controls factors such as decrease in body weight, glomerular hyperfiltration, and hypertension. As such, SGLT2 inhibitors may increase glycemic control without causing these side effects. ${ }^{5}$

To date, SGLT2 inhibitors that are currently approved in at least one major market (eg, the United States, the European Union, and Japan) include canagliflozin, dapagliflozin, empagliflozin, luseogliflozin, ipragliflozin, and tofogliflozin. ${ }^{6-8}$ However, large trials evaluating the efficacy, effect on body weight, cardiac parameters, renal parameters, and safety of SGLT2 inhibitors are lacking. In addition, recent research has raised concerns that the use of SGLT2 inhibitors is associated with an increase in diabetic ketoacidosis. ${ }^{9}$ Therefore, we conducted this meta-analysis to investigate the association between SGLT2 inhibitor monotherapy and diabetic ketoacidosis. We also evaluated the safety of SGLT2 inhibitor monotherapy and its effects on weight loss as well as on glycemic and other clinical laboratory parameters in T2DM patients.

\section{Materials and methods Information sources and search strategy}

PubMed and MEDLINE were searched for randomized controlled trials in which the efficacy, safety, and effect on body weight of luseogliflozin, canagliflozin, dapagliflozin, empagliflozin, ipragliflozin, remogliflozin, and tofogliflozin versus placebo in type 2 diabetic patients had been assessed. For specific trials on SGLT2 inhibitor agents, the generic names variably combined with "efficacy", "safety", "weight loss", and "diabetic ketoacidosis" were used as search keywords. The publication cutoff date for these articles was set as December 2015. The titles and abstracts of the identified trials were further analyzed, and those that did not meet the eligibility criteria were excluded.

\section{Eligibility criteria}

Full-text randomized trials published in English and conducted on T2DM patients $>18$ years old were eligible. It was required that articles should have assessed the efficacy and safety of SGLT2 inhibitors (luseogliflozin, canagliflozin, dapagliflozin, empagliflozin, ipragliflozin, remogliflozin, or tofogliflozin) as monotherapy versus placebo therapy, and data for at least 12 weeks of intervention should have been available. The methodology should have been described, and articles should have included at least one of the following variables: HbA1c, fasting plasma glucose (FPG), 2-hour postprandial glucose (2-hour PPG), renal parameters, cardiac parameters, ketone bodies, or adverse events. Trials that included other treatments (eg, thiazolidinediones or biguanides) as comparators in addition to placebos were eligible. Outcome measures of SGLT2 inhibitors were compared separately with these treatments. Articles that were not randomized trials, those in which SGLT2 inhibitors were not compared with placebos, those with incomplete methodology, those that used SGLT2 inhibitors as combination therapy, those for which full text was not available, and trials using animals or healthy human subjects were excluded from this meta-analysis.

\section{Study selection and quality assessment}

Two authors independently reviewed the abstracts to determine the eligibility of the articles. They then compared the text of the articles with the eligibility criteria to determine whether to include them, and disagreements were resolved through mutual consensus.

The Cochrane Collaboration Risk of Bias Tool was used to assess the quality of the trials and to determine bias. ${ }^{10}$ The scale consists of six categories: selection bias, performance 
bias, detection bias, attrition bias, reporting bias, and other bias. These six categories are further subdivided into seven subcategories. Selection bias comprises random sequence generation and allocation concealment, performance bias consists of blinding of participants and personnel, detection bias consists of blinding of the outcome assessment, attrition bias consists of incomplete outcome data, reporting bias consists of selective reporting, and other bias includes anything else ideally prespecified. Each variable is scored as low, high, or unclear.

\section{Summary measures}

The efficacy measures included HbAlc, FPG, and 2-hour PPG evaluated for glycemic efficacy for the groups receiving SGLT2 inhibitors versus those receiving other antidiabetic drugs (active control) or placebos. Similarly, the changes in weight (in kilograms), renal parameters, cardiac parameters, and blood level of ketone bodies were evaluated for patients receiving SGLT2 inhibitors therapy versus those receiving active control or placebo. The measures of safety included adverse events reports of patients receiving SGLT2 inhibitors, placebo therapy, or active control.

\section{Data synthesis}

All statistical analyses were performed with RevMan (v5.3; Cochrane collaboration). All end points for patients on SGLT2 inhibitors were compared with those of patients on placebo therapy and active control. The mean difference and its 95\% two-sided confidence interval (CI) were applied for continuous variables, whereas the risk ratios and their $95 \%$ two-sided CIs were used for dichotomous outcomes. All $P$-values were two-tailed, and $P \leq 0.05$ was considered as statistically significant for all analyses. Calculation of effect sizes for continuous variables, assessments of heterogeneity, imputation of missing data, and assessments of publication bias were also performed.

Statistical heterogeneity across trials was assessed by the Cochran's $Q$ statistic and $I^{2}$ tests. ${ }^{11}$ If heterogeneity was found, a random-effects model that included weighting of the trials was used $(P<0.10)$. If heterogeneity was not found, a fixed-effects model with weighting of the trials was used.

\section{Results}

A total of 1,856 titles were retrieved from the databases (Figure 1). After 1,268 were determined to be ineligible on the basis of the title, 588 articles were identified for abstract screening. A total of 558 articles were excluded on the basis of the content of their abstracts. Thirty articles were identified for full-text screening. Of these, 13 trials were determined to be eligible and were included in the analysis. ${ }^{12-24}$

The characteristics of all the eligible trials are included in Table 1. For the comparison of SGLT2 inhibitors versus placebo therapy, the efficacy and safety of dapagliflozin versus placebo were reported in four trials. ${ }^{12-15}$ Luseogliflozin and canagliflozin were compared with placebo in three trials each. ${ }^{16-21}$ Ipragliflozin, tofogliflozin, and remogliflozin were compared with placebo in one trial each. ${ }^{22-24}$ Active control was compared with SGLT2 inhibitor therapy in three trials. ${ }^{15,22,24}$ In two trials, the active control was metformin, and in one trial, it was pioglitazone. . $^{1,22,24}$ Not all outcomes were reported in all trials. For glycemic efficacy, the mean changes from baseline in $\mathrm{HbA} 1 \mathrm{c}$ and FPG were reported in ten trials, and the change from baseline in 2-hour PPG was reported in six trials. ${ }^{12-16,18-24}$ Changes from baseline in weight were reported in eleven trials. ${ }^{12-24}$ Changes in lipid parameters were reported in eight trials. ${ }^{13,16-21,23}$ Changes in high-density lipoprotein (HDL) levels and low-density lipoprotein (LDL) levels were reported in seven trials. ${ }^{13,16-18,20,21,23}$ Changes in triglycerides were reported in eight trials, and changes in total cholesterol and LDL/HDL ratio were reported in four trials. ${ }^{13,16-21,23}$ For renal parameters, changes in estimated glomerular filtration rate (eGFR), the albumin-to-creatinine ratio, and albumin were reported in one trial each. ${ }^{12,15,17,23}$ Changes in creatinine were reported in seven trials, changes in uric acid were reported in six trials, and changes in blood urea nitrogen (BUN) were reported in eight trials. ${ }^{12-16,18-21,23}$ Changes in ketones were reported in four trials. ${ }^{17,20,21,23}$ In two trials each, changes in total ketone bodies, acetoacetic acid, and $\beta$-hydroxybutyric acid were assessed. ${ }^{17,20,21,23}$

\section{Risk of bias of included trials}

Overall, most trials had a low risk of bias in at least two of the variables assessed (Figure 2). Common issues found during the assessment of quality were inadequate descriptions of blinding of outcome assessment (eleven trials), inadequate descriptions of the randomization sequence (ten trials), inadequate descriptions of the allocation concealment or the blinding of participants or personnel (eight trials for each variable), and discrepancies between the variables mentioned in the methodology section and those reported in the results section (two trials).

\section{Change in $\mathrm{HbAlc}$}

For the comparison between SGLT2 inhibitors and placebo therapy, a total of ten trials were included in the pooled analysis $(2,809$ patients). Overall, statistically significant treatment 
Results from Medline

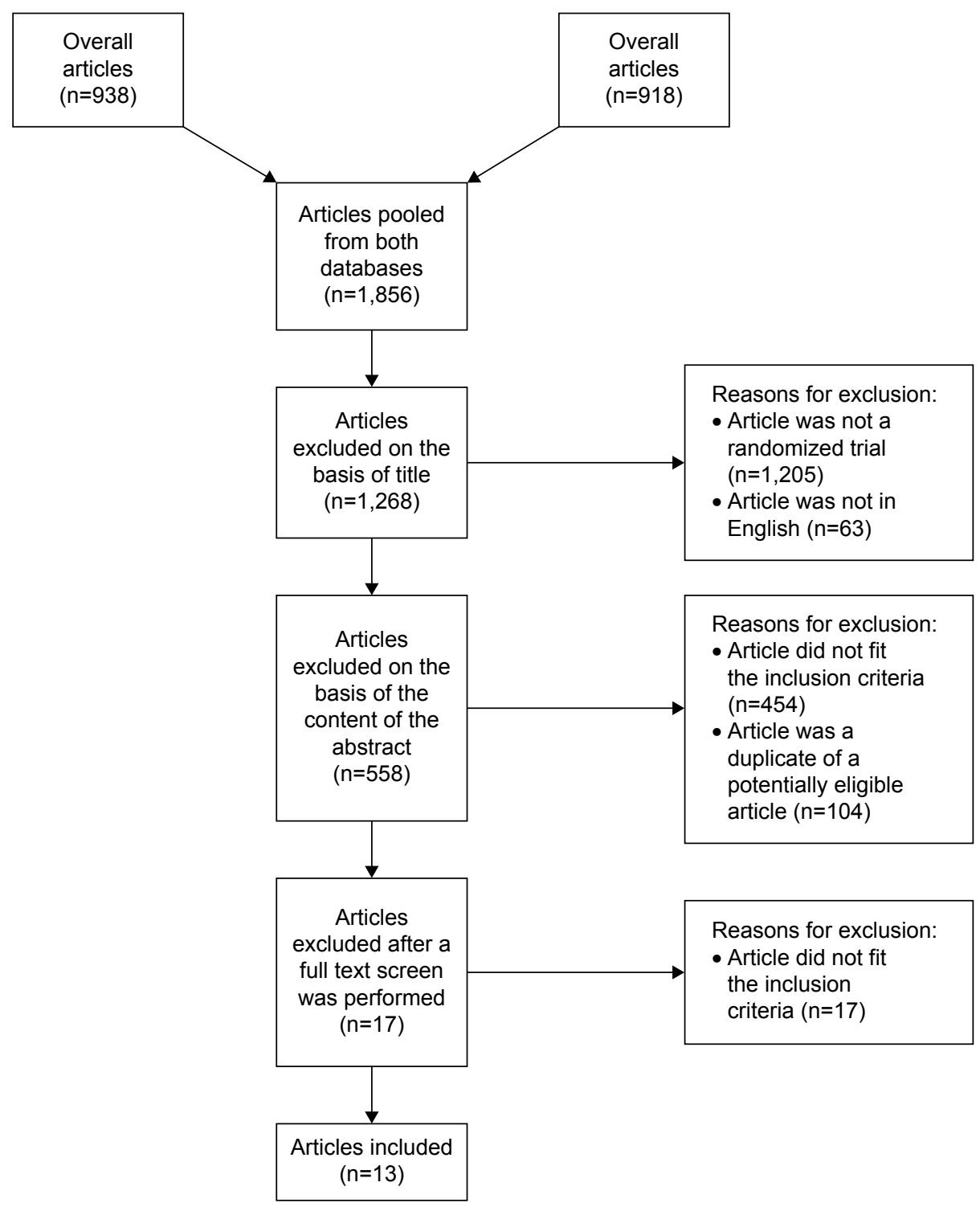

Figure I PRISMA diagram.

effects favored SGLT2 inhibitors (mean difference $=-3.35$, [95\% CI, -4.32, -2.39], $P<0.001)$. Heterogeneity as assessed by $I^{2}$ was $99 \%$ (Figure $3 \mathrm{~A}$ ).

In three trials ( 850 participants), changes from baseline in $\mathrm{HbA1c}$ for SGLT inhibitors versus active control were reported. No significant difference was found for patients receiving SGLT2 inhibitor treatment compared with those receiving active control $(P=0.60)$. Heterogeneity as assessed by $I^{2}$ was $0 \%$ (Figure $3 \mathrm{~B}$ ).

\section{Change in FPG}

Changes in FPG for SGLT2 inhibitors as compared with placebo therapy and with active control are shown in Figure 4A and $\mathrm{B}$. Ten trials $(2,809$ participants $)$ reported changes from baseline in FPG for SGLT2 inhibitors compared with placebo therapy, and three trials ( 850 participants) reported changes from baseline in FPG for SGLT2 inhibitors versus active control. Although treatment effects favored SGLT2 inhibitors compared with both placebo therapy and active control, the results were not statistically significant $(P=0.07$ for SGLT2 inhibitors vs placebo therapy and $P=0.6$ for SGLT2 inhibitors vs active control). For the comparisons of SGLT2 inhibitors versus placebo therapy and SGLT2 inhibitors versus active control, heterogeneity as assessed by $I^{2}$ was $0 \%$.

\section{Change in 2-hour PPG}

Changes from baseline in 2-hour PPG for SGLT2 inhibitors versus placebo therapy are shown in Figure 5. Six trials 


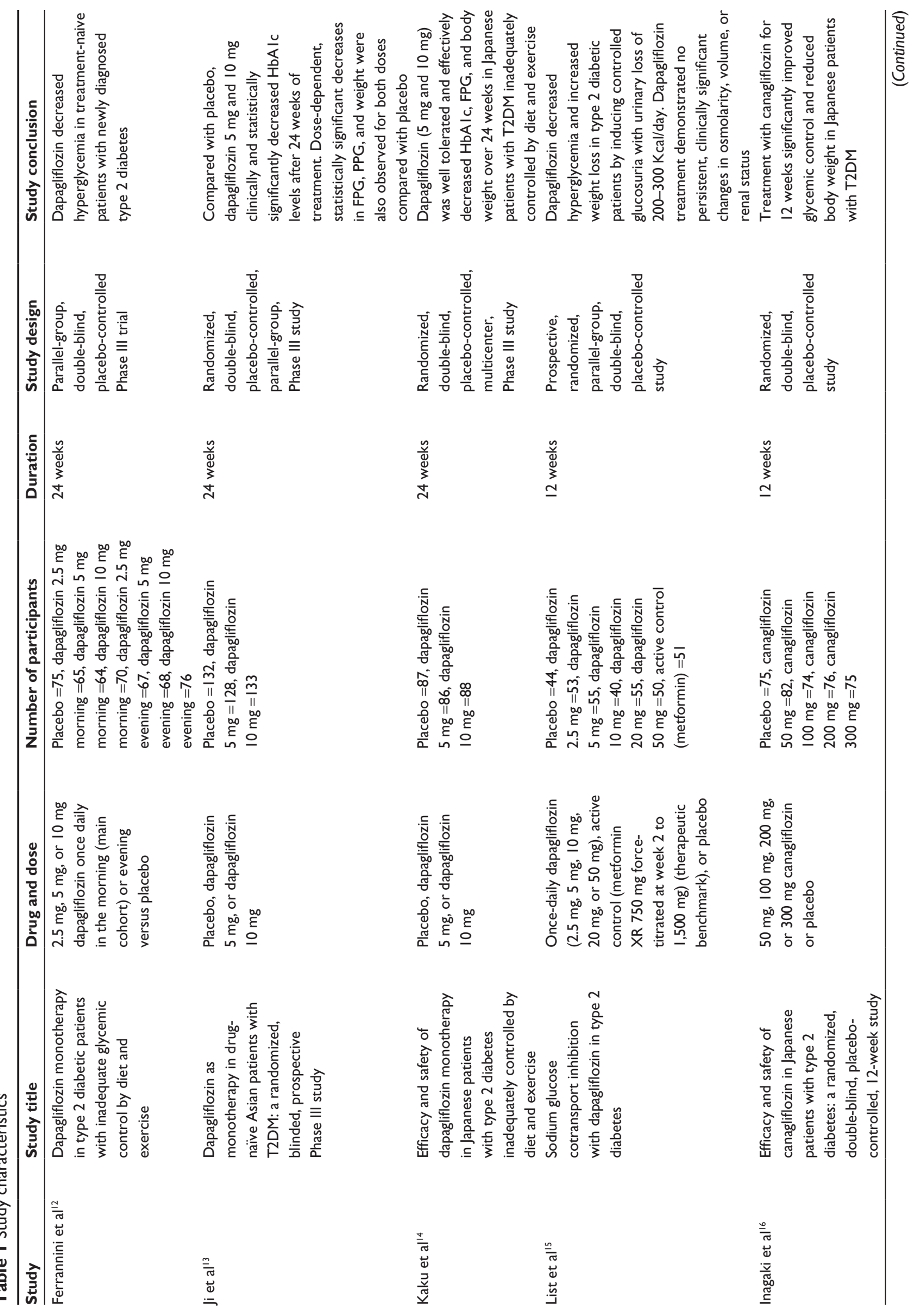



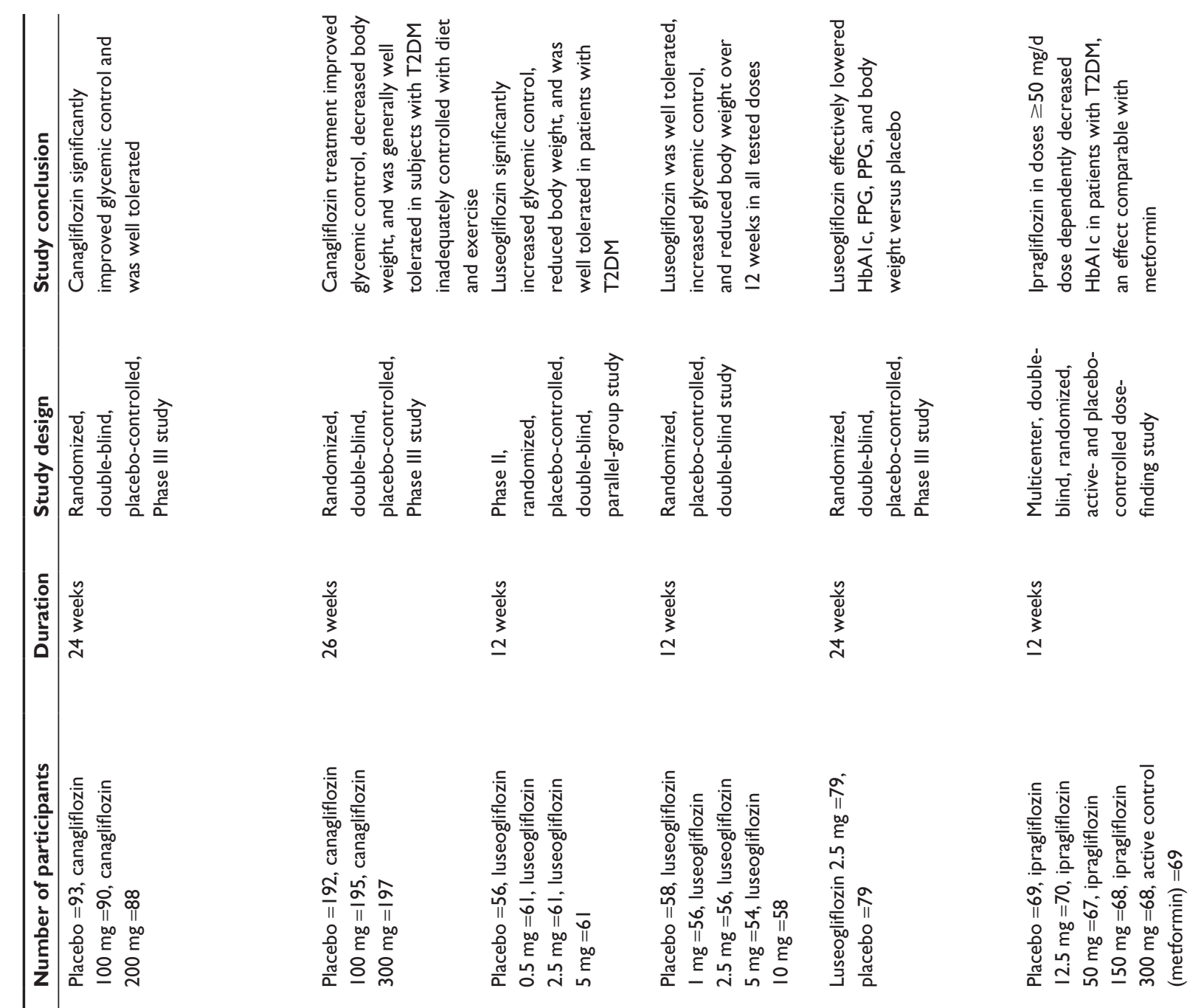

\begin{tabular}{|c|c|c|c|c|c|}
\hline 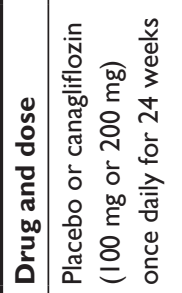 & 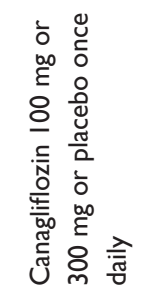 & 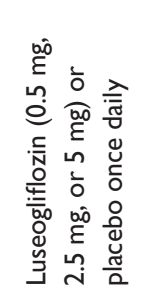 &  & 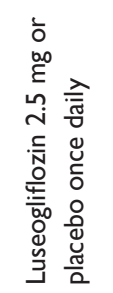 &  \\
\hline
\end{tabular}

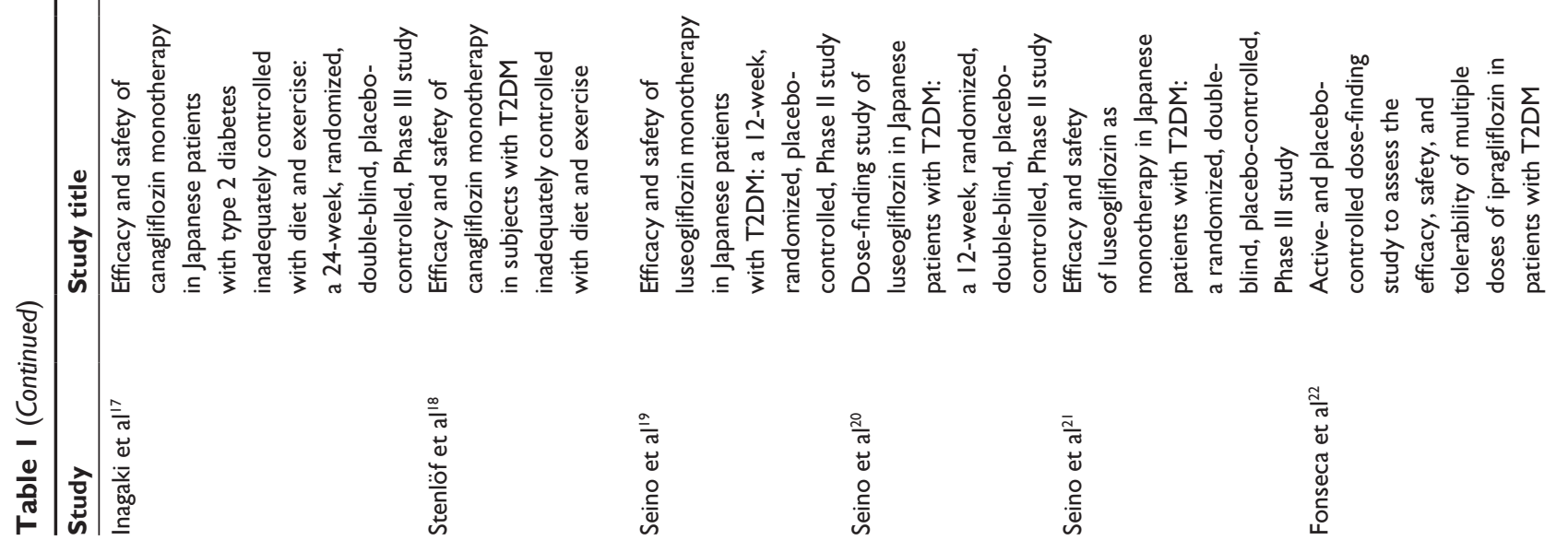





$\frac{y}{d}$
0
$\vdots$
$\vdots$
$d$




(1,558 participants) reported changes from baseline in 2-hour PPG for SGLT2 inhibitors versus placebo therapy, and no trials reported changes from baseline in 2-hour PPG for SGLT2 inhibitors versus active control. Treatment effects that favored SGLT2 inhibitors over placebo therapy were not statistically significant $(P=0.64)$. Heterogeneity as assessed by $I^{2}$ was $0 \%$.

\section{Change in weight}

For the comparison between SGLT2 inhibitors and placebo therapy, eleven trials were included in the pooled analysis (3,993 patients). Three trials ( 850 participants) were included in the pooled analysis for SGLT2 inhibitors versus active control. Changes from baseline in weight for SGLT2 inhibitors versus placebo therapy are shown in Figure 6A. Weight significantly decreased for SGLT2 inhibitors versus placebo $(-0.32,[95 \% \mathrm{CI},-0.48,-0.17], P<0.001)$. By contrast, the decreases in weight in the SGLT2 inhibitor groups versus the active control groups were not statistically significant $(-10$, [95\% CI, $-0.27,0.07], P=0.25$ ) (Figure 6B). Heterogeneity as assessed by $I^{2}$ for SGLT 2 inhibitors versus placebo therapy and active control was $74 \%$ and $0 \%$, respectively.

\section{Change in lipid parameters}

Changes in total cholesterol, HDL, LDL, and triglycerides for SGLT2 inhibitors versus active control were not reported in any of the eligible trials. However, for the comparisons of SGLT2 inhibitors versus placebo therapy, four trials $(1,153$ participants) included changes from baseline in total cholesterol, seven trials (2,288 participants) included changes from baseline in HDL and LDL, and eight trials (2,524 participants) included changes from baseline in triglycerides as outcomes. No statistically significant changes occurred in patients receiving SGLT2 inhibitors versus placebo therapy (Table 2). Heterogeneity as assessed by $I^{2}$ was $0 \%$ for each of these variables.

Changes from baseline in LDL/HDL ratios for SGLT2 inhibitors compared with placebo and with active control are found in Table 2. Changes from baseline in LDL/HDL ratios for SGLT2 inhibitors versus placebo were reported in four trials (1,409 participants), and in one trial (173 participants), the change from baseline in the LDL/HDL ratio for SGLT2 inhibitors versus active control was reported. However, no statistically significant changes occurred when SGLT2 inhibitors were compared with active control $(P=0.65)$. The statistically significant decreases favored SGLT2 inhibitors versus placebo $(-0.30,[95 \% \mathrm{CI}, 0.57,0.02], P=0.03)$ with a heterogeneity of $79 \%$. 


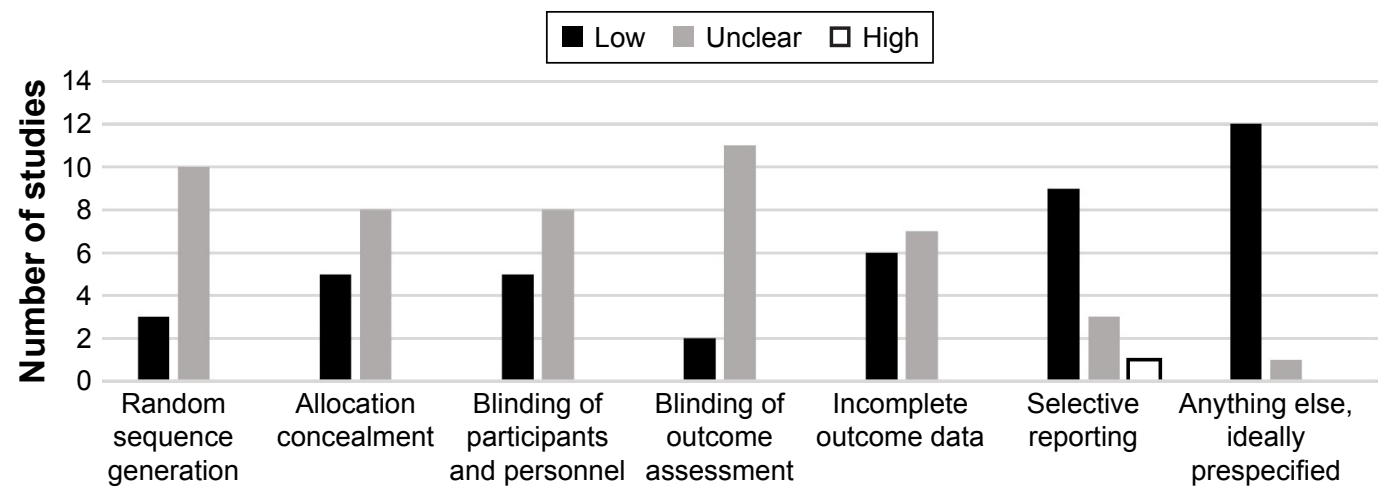

Assessment category

Figure 2 Assessment of study quality and bias.

\section{Change in renal parameters}

Changes from baseline in BUN, eGFR, serum creatinine, uric acid, albumin, and the albumin-to-creatinine ratio for SGLT2 inhibitors versus placebo are shown in Table 3. Eight trials (2,567 participants) reported changes from baseline in BUN, two trials (2,234 participants) reported changes from baseline in serum creatinine, and six trials (1,712 participants) reported changes from baseline in uric acid. One study each reported a change from baseline in eGFR, albumin, and the albumin-to-creatinine ratio (220, 485 , and 280 participants, respectively). Statistically significant increases in serum creatinine occurred among patients receiving SGLT2 inhibitors versus those receiving placebo therapy $(0.70,[95 \% \mathrm{CI}, 0.03,1.37], P=0.04)$. Patients receiving SGLT2 inhibitors experienced a moderate decrease in uric acid compared with patients receiving placebo therapy $(-0.73$ [95\% CI $-1.24,-0.21], P=0.005)$ with a heterogeneity of $94 \%$. No statistically significant changes occurred for eGFR, BUN, albumin levels, and the albumin-to-creatinine ratio. Heterogeneity for the eGFR, albumin levels, and the albumin-to-creatinine ratio was not applicable because only one study each reported these variables. ${ }^{12,17,23}$ Heterogeneity for BUN was $0 \%$ as assessed by $I^{2}$.

\begin{tabular}{|c|c|c|c|c|c|c|}
\hline $\begin{array}{l}\text { Study or } \\
\text { subgroup }\end{array}$ & $\begin{array}{l}\text { SGLT2 inh } \\
\text { Mean }\end{array}$ & $\begin{array}{l}\text { bitors } \\
\text { SD }\end{array}$ & Total & $\begin{array}{l}\text { Placebo } \\
\text { Mean }\end{array}$ & SD & Total \\
\hline Ferrannini et al ${ }^{12}$ & -0.76627 & 0.799905 & 410 & -0.23 & 0.75 & 75 \\
\hline Inagaki et al ${ }^{17}$ & -0.74989 & 0.433792 & 178 & 0.29 & 0.4557 & 93 \\
\hline $\mathrm{Ji}$ et $\mathrm{al}^{13}$ & -1.07567 & 0.617273 & 261 & -0.29 & 0.626223 & 132 \\
\hline Kaku et al ${ }^{14}$ & -0.89467 & 0.361697 & 165 & -0.028 & 0.37027 & 55 \\
\hline Kaku et $\mathrm{al}^{23}$ & -0.43023 & 0.315627 & 174 & -0.06 & 0.317013 & 87 \\
\hline List et al ${ }^{15}$ & -0.73996 & 0.02416 & 279 & -0.18 & 0.01 & 54 \\
\hline Seino et al ${ }^{19}$ & -0.57786 & 0.363641 & 182 & 0.06 & 0.302012 & 54 \\
\hline Seino et $\mathrm{al}^{20}$ & -0.39269 & 0.197013 & 223 & 0.22 & 0.204537 & 57 \\
\hline Seino et $a^{21}$ & -0.63 & 0.542649 & 79 & 0.13 & 0.542649 & 79 \\
\hline Sykes et $\mathrm{al}^{24}$ & -0.66281 & 0.687316 & 139 & -0.19 & 0.6468 & 33 \\
\hline \multicolumn{3}{|c|}{ Total $(95 \% \mathrm{Cl})$} & \multicolumn{2}{|l|}{2,090} & & 719 \\
\hline \multirow{2}{*}{\multicolumn{7}{|c|}{ Heterogeneity: $\chi^{2}=749.20, d f=9(P<0.00001) ; l^{2}=99 \%$}} \\
\hline est for overall & $: Z=29.8$ & & & & & \\
\hline
\end{tabular}

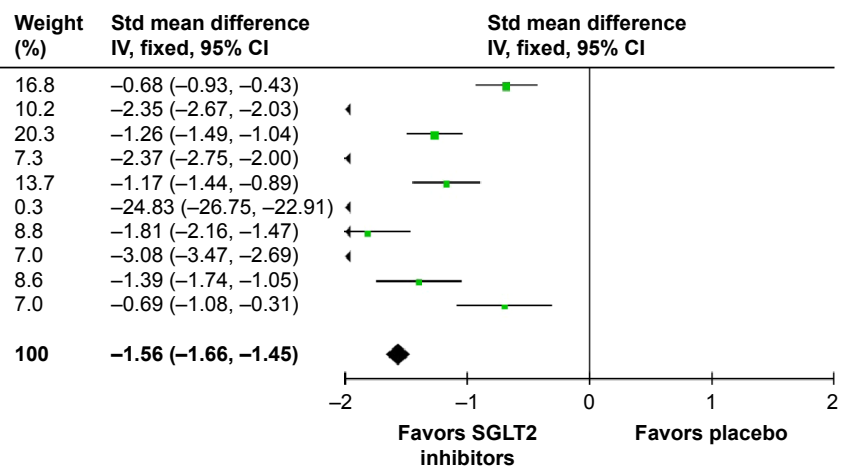

\begin{tabular}{|c|c|c|c|c|c|}
\hline \multirow{2}{*}{$\begin{array}{l}\text { Study or } \\
\text { subgroup }\end{array}$} & \multicolumn{2}{|c|}{ SGLT2 inhibitors } & \multirow[b]{2}{*}{ Total } & \multicolumn{2}{|c|}{ Active control } \\
\hline & Mean & SD & & Mean & SD \\
\hline $\begin{array}{l}\text { Fonseca et } \mathrm{al}^{22} \\
\text { List et } \mathrm{al}^{15} \\
\text { Sykes et } \mathrm{al}^{24}\end{array}$ & $\begin{array}{l}-1.22762 \\
-22.1685 \\
-1.12597\end{array}$ & $\begin{array}{l}5.84442 \\
36.85281 \\
68.6002\end{array}$ & $\begin{array}{l}273 \\
279 \\
139\end{array}$ & $\begin{array}{l}-1.18 \\
-18 \\
-1.66\end{array}$ & $\begin{array}{l}6.134696 \\
9 \\
141.4944\end{array}$ \\
\hline \multicolumn{4}{|c|}{$\begin{array}{l}\text { Total }(95 \% \mathrm{Cl}) \\
\text { Heterogeneity: } \chi^{2}=0.43, d f=2(P=0.81) ; I^{2}=0 \% \\
\text { Test for overall effect: } Z=0.53(P=0.60)\end{array}$} & & \\
\hline
\end{tabular}

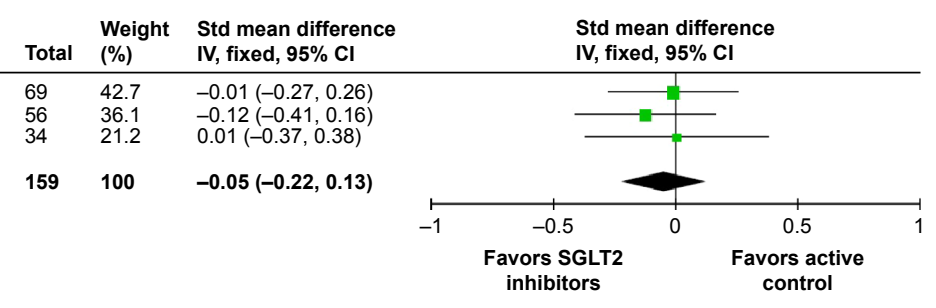

Figure 3 Changes from baseline in HbAlc for patients treated with SGLT2 inhibitors versus placebo or active control.

Notes: (A) SGLT2 inhibitors compared with placebo. (B) SGLT2 inhibitors compared with active control.

Abbreviations: HbAlc, glycated hemoglobin; Cl, confidence interval; $d f$, degrees of freedom; SGLT2, sodium glucose cotransporter 2; Std, standard; SD, standard deivation. 




Figure 4 Changes from baseline in FPG for patients treated with SGLT2 inhibitors versus placebo or active control.

Notes: (A) SGLT2 inhibitors compared with placebo. (B) SGLT2 inhibitors compared with active control.

Abbreviations: FPG, fasting plasma glucose; Cl, confidence interval; df, degrees of freedom; SGLT2, sodium glucose cotransporter 2; Std, standard; SD, standard deviation.

Changes from baseline in BUN and uric acid for SGLT2 inhibitors versus active control are shown in Table 3. The eGFR, albumin levels, and the albumin-to-creatinine ratio were not reported in trials in which SGLT2 inhibitors were compared with active control; however, in one trial (335 participants), changes from baseline in BUN and uric acid were reported for these treatments. ${ }^{15}$

For uric acid (Table 3), statistically significant decreases occurred for patients receiving SGLT2 inhibitors versus active control $(-1.28$, [95\% CI, $-1.58,-0.98], P<0.001)$. However, no statistically significant changes occurred for BUN (Table 3). Heterogeneity for BUN and uric acid was not applicable because only one study reported these variables (List et $\mathrm{al}^{15}$ ).

\section{Change in ketone bodies}

Changes in acetoacetic acid, $\beta$-hydroxybutyric acid, and total ketone bodies for SGLT2 inhibitors compared with placebo therapy are shown in Figure 7. Changes in ketone levels were not assessed in any of the trials in which SGLT2 inhibitors were compared with active control. However, for the comparison of SGLT2 inhibitors versus placebo therapy, three trials (658 participants) reported changes from baseline in acetoacetic acid and $\beta$-hydroxybutyric acid, and two trials (491 participants) reported changes from baseline in levels of total ketones. No statistically significant changes occurred for any of these variables $(P=0.93)$. Heterogeneity for acetoacetic acid, $\beta$-hydroxybutyric acid, and total ketone bodies was $0 \%$.

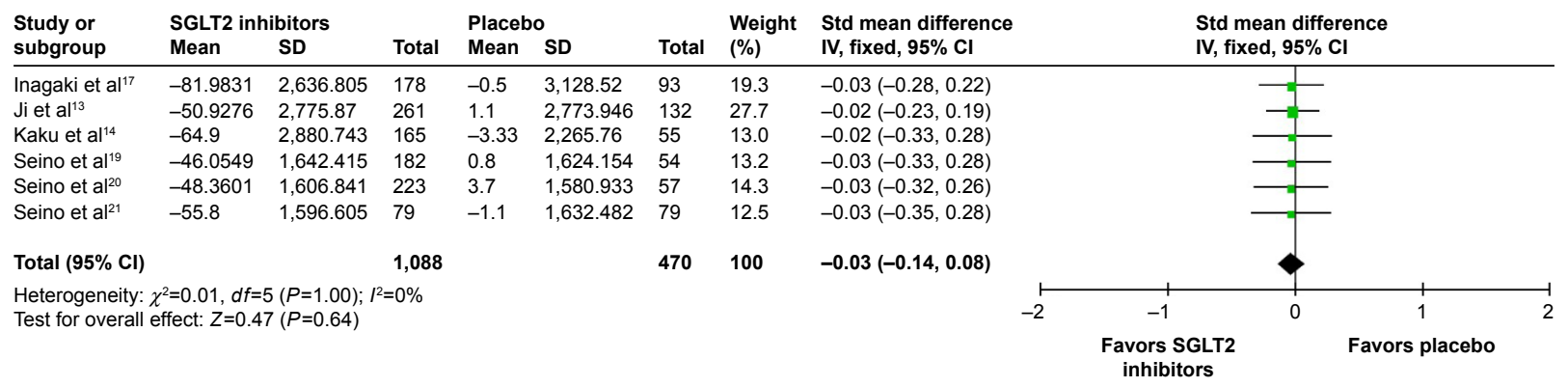

Figure 5 Changes from baseline in 2-hour PPG for patients treated with SGLT2 inhibitors versus placebo.

Abbreviations: PPG, postprandial glucose; Cl, confidence interval; $d f$, degrees of freedom; SGLT2, sodium glucose cotransporter 2; Std, standard; SD, standard deviation. 


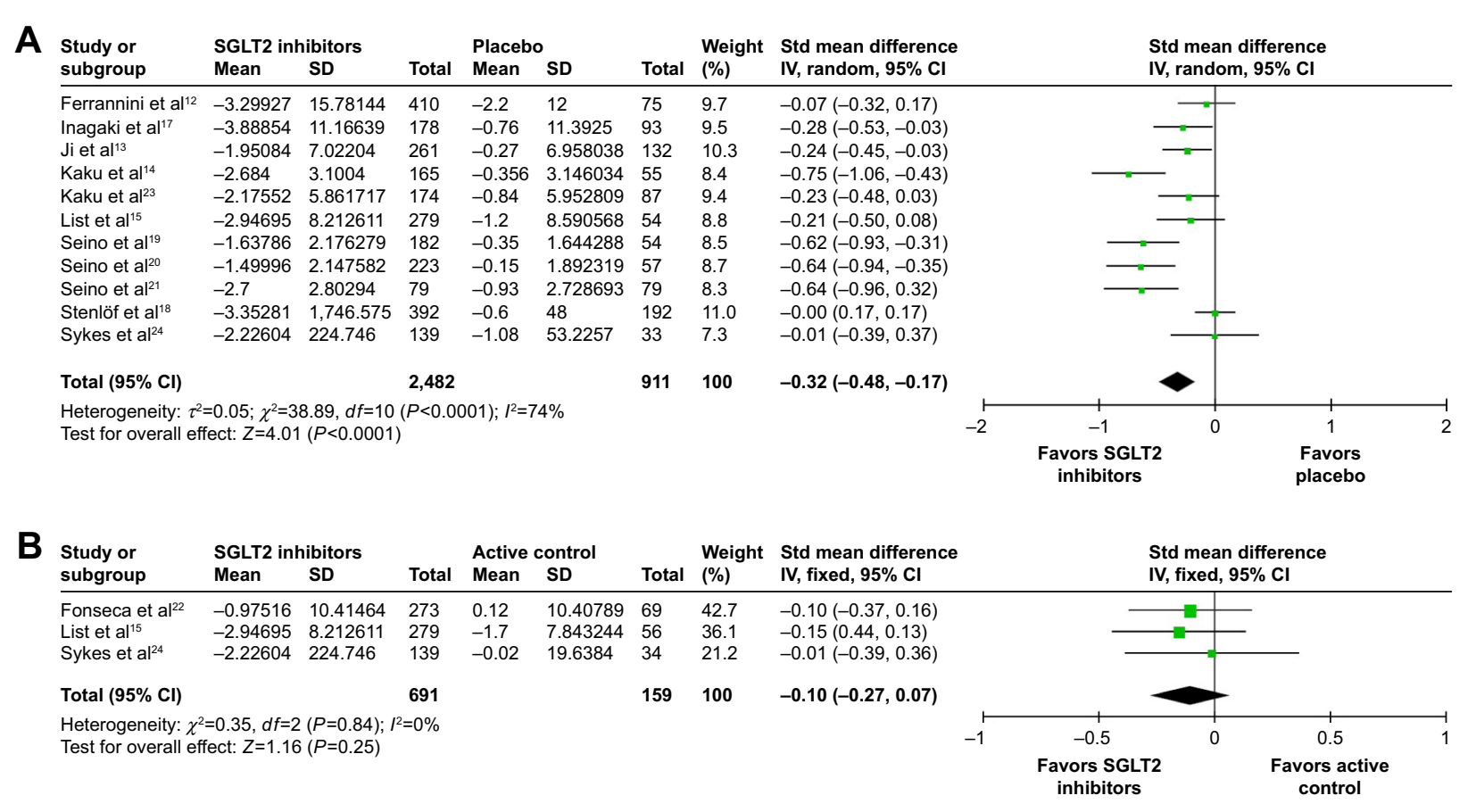

Figure 6 Changes from baseline in weight for patients treated with SGLT2 inhibitors versus placebo or active control.

Notes: (A) SGLT2 inhibitors compared with placebo. (B) SGLT2 inhibitors compared with active control.

Abbreviations: $\mathrm{Cl}$, confidence interval; $d f$, degrees of freedom; SGLT2, sodium glucose cotransporter 2; Std, standard; SD, standard deviation.

Table 2 Serum lipid level

\begin{tabular}{llllll}
\hline Variable & Comparator & $\begin{array}{l}\text { Mean } \\
\text { difference }\end{array}$ & $\begin{array}{l}\text { Cl lower } \\
\text { bound }\end{array}$ & $\begin{array}{l}\text { Cl upper } \\
\text { bound }\end{array}$ \\
\hline HDL & Placebo & 0.05 & -0.04 & 0.14 & 0.32 \\
LDL & Placebo & 0.01 & -0.08 & 0.10 & 0.83 \\
LDL/HDL ratio & Placebo & -0.30 & -0.57 & -0.02 & 0.03 \\
LDL/HDL ratio & Active & -0.09 & -0.46 & 0.29 & 0.65 \\
TG & Placebo & -0.00 & -0.09 & 0.08 & 0.94 \\
TC & Placebo & 0.01 & -0.12 & 0.14 & 0.85 \\
\hline
\end{tabular}

Abbreviations: $\mathrm{Cl}$, confidence interval; HDL, high-density lipoprotein; LDL, low-density lipoprotein; TG, triglyceride; TC, total cholesterol.

Table 3 Renal profile

\begin{tabular}{llllll}
\hline Variable & Comparator & $\begin{array}{l}\text { Mean } \\
\text { difference }\end{array}$ & $\begin{array}{l}\text { Cl lower } \\
\text { bound }\end{array}$ & $\begin{array}{l}\text { Cl upper } \\
\text { bound }\end{array}$ & $\begin{array}{l}\text { P-value } \\
\text { Serum creatinine }\end{array}$ \\
Uric acid & Placebo & 0.70 & 0.03 & 1.37 & 0.04 \\
Uric acid & Placebo & -0.73 & -1.24 & -0.21 & 0.005 \\
Albumin & Active & -1.28 & -1.58 & -0.98 & $<0.001$ \\
Albumin/creatinine ratio & Placebo & -0.01 & -0.26 & 0.24 & 0.94 \\
BUN & Placebo & -0.00 & -0.25 & 0.25 & 0.16 \\
BUN & Placebo & 0.07 & -0.02 & 0.46 & 0.11 \\
eGFR & Active & 0.18 & -0.11 & 0.28 & 0.23 \\
\hline
\end{tabular}

Abbreviations: $\mathrm{Cl}$, confidence interval; BUN, blood urea nitrogen; eGFR, estimated glomerular filtration rate. 


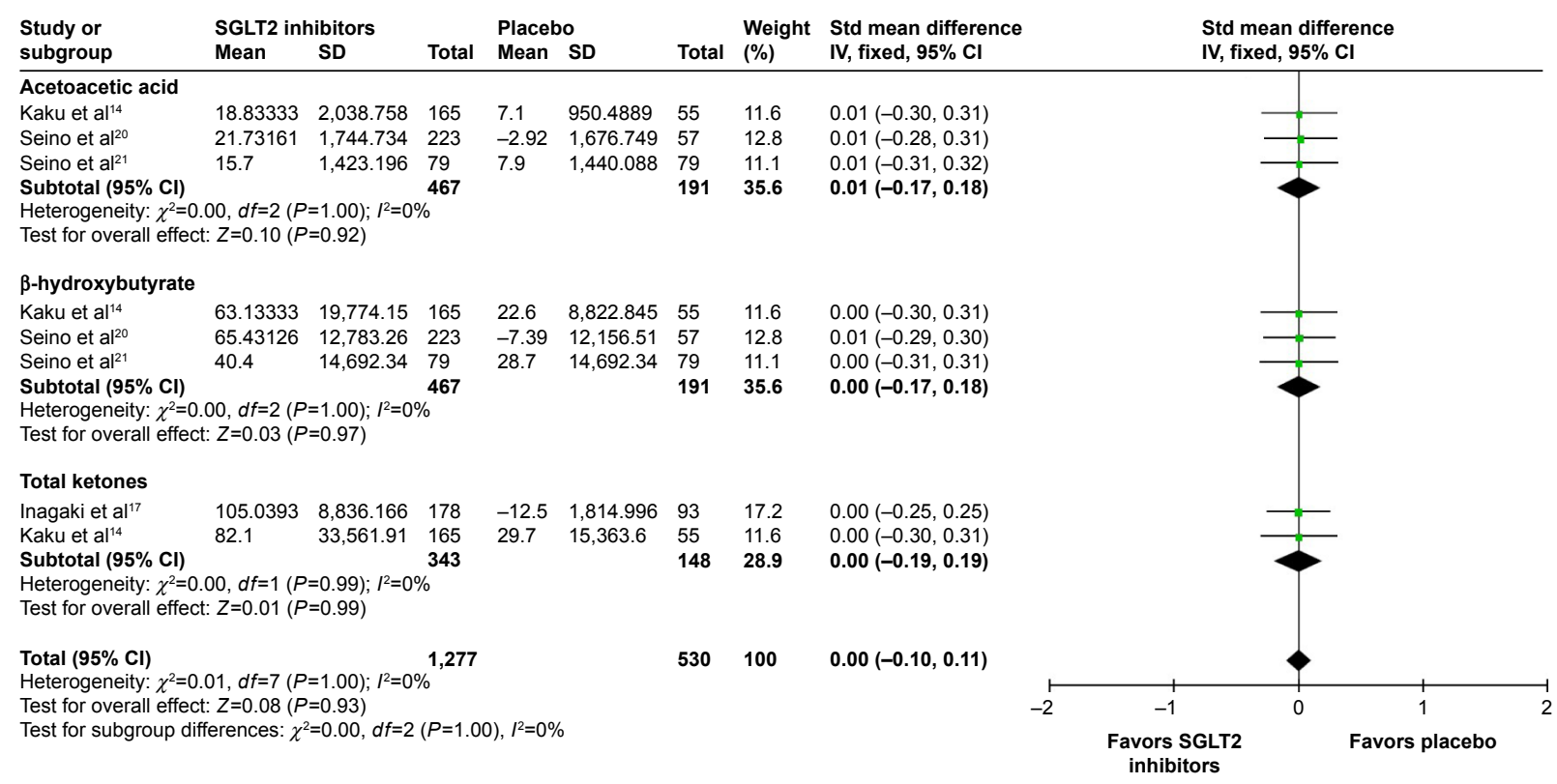

Figure 7 Changes from baseline in ketones for patients treated with SGLT2 inhibitors versus placebo.

Abbreviations: $\mathrm{Cl}$, confidence interval; $d f$, degrees of freedom; SGLT2, sodium glucose cotransporter 2; Std, standard; SD, standard deviation.

\section{Overall adverse events}

Risk ratios for overall adverse events are shown in Table 4. For overall adverse events, patients treated with SGLT2 inhibitors had a greater risk of experiencing an adverse event than patients treated with placebos $(1.08,[95 \% \mathrm{CI}, 1.01,1.16]$, $P=0.02)$. Similarly, for adverse events related to treatment, the relative risk significantly increased in the SGLT2 inhibitor group as compared to that of placebo groups (1.52, [95\% CI, $1.14,2.02], P=0.004)$. No statistically significant treatment effects occurred for discontinuations due to adverse events, deaths, and serious adverse events. Heterogeneity for the overall adverse events and adverse events related to treatment was $34.2 \%$ and $76.7 \%$, respectively, whereas it was $0 \%$ for serious adverse events, discontinuations due to adverse events, and deaths.

No significant difference in risk was found for SGLT2 inhibitors versus active control for overall adverse events, discontinuations due to adverse events, serious adverse events, and adverse events related to treatment. Heterogeneity for the overall adverse events was $29.6 \%$, whereas it was $0 \%$ for adverse events related to treatment, serious adverse events, and discontinuations due to adverse events.

\section{Adverse events by preferred term}

Risk ratios for adverse events by preferred term for SGLT2 inhibitors versus placebo therapy are included in Table 5 . Overall, the only two adverse events for which the risk was significantly greater for patients in either group were increased blood ketone bodies and diarrhea. For increased blood ketone bodies (two trials; 602 participants), the risk was greater for patients receiving SGLT2 inhibitors compared with patients receiving placebo (relative risk $=3.80$, [95\% CI, 1.20, 12.00], $P=0.02$ ). Patients receiving SGLT2 inhibitors experienced a lower risk of diarrhea than those receiving placebo (relative risk $=1.09,[95 \% \mathrm{CI}, 0.57$, 2.09], $P=0.80$ ).

Risk ratios for adverse events by preferred term for SGLT2 inhibitors versus active control are included in Table 5. The adverse events that presented a significant risk for patients in either group were diarrhea, rash, and tendonitis. The risk of diarrhea was greater for patients receiving active control than those receiving SGLT2 inhibitors $(0.33$, [95\% CI, 0.14, 0.79], $P=0.01)$. Similarly, the risk of rash and tendonitis was greater for patients receiving active control than those receiving SGLT2 inhibitors $(P=0.05)$. In the comparison of adverse events by the preferred term between SGLT2 inhibitors and placebo therapy or active control, no obvious heterogeneity was found within the trials $(I \leq 45 \% ; P>0.05$ for all adverse events).

\section{Adverse events of special interest}

Adverse events of special interest are shown in Table 6. For events suggestive of urinary tract infection (eight trials; 3,253 participants), patients receiving SGLT2 inhibitors experienced a greater risk compared with patients receiving placebos (relative risk $=1.57$, [95\% CI, 1.04, 2.36], $P=0.03$ ). 

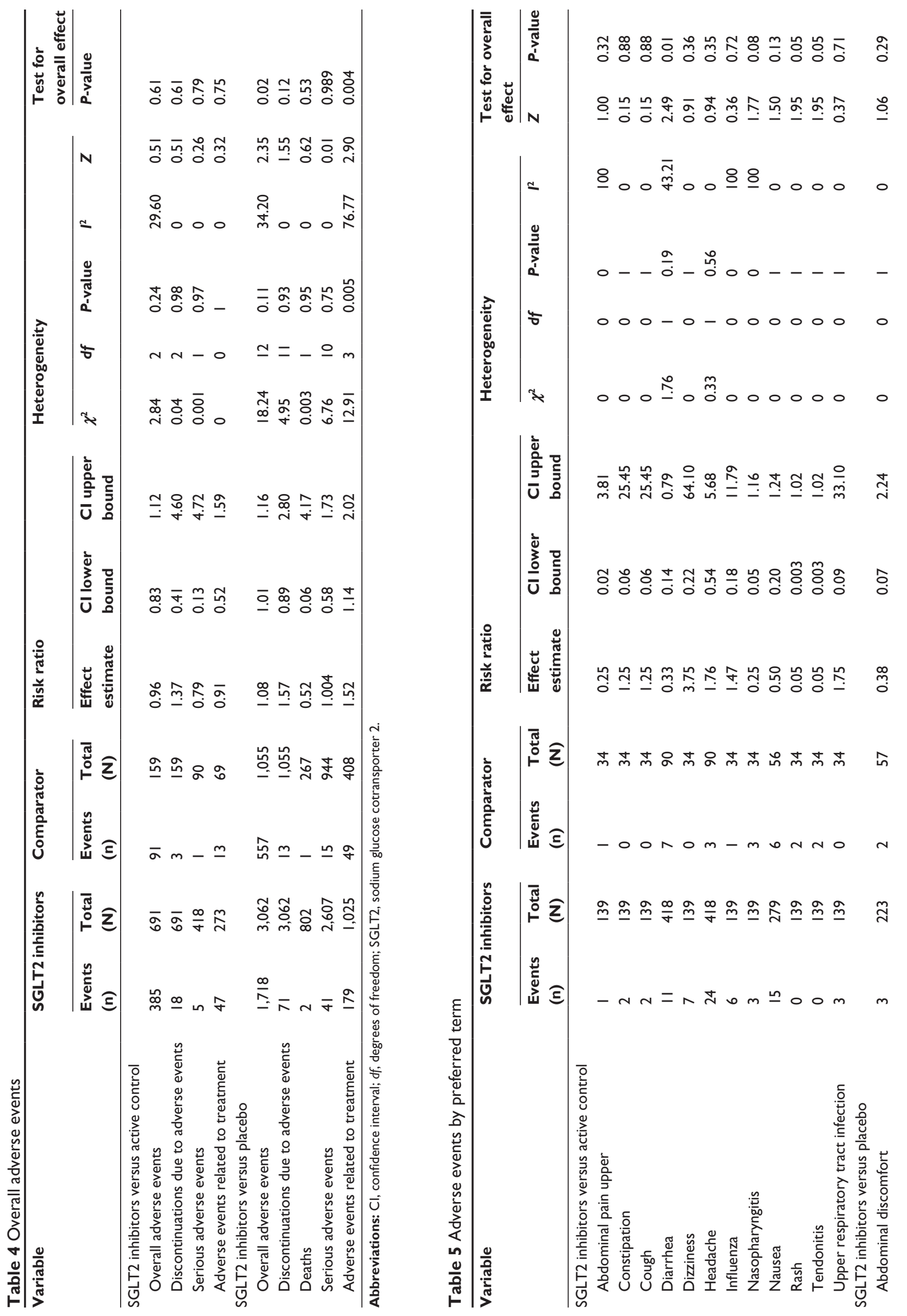






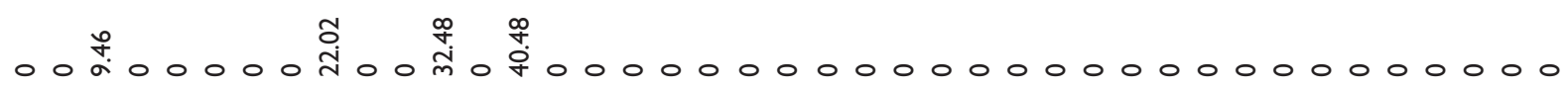

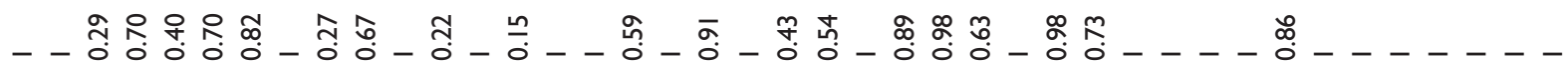

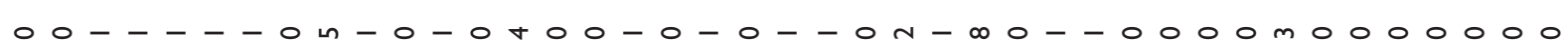

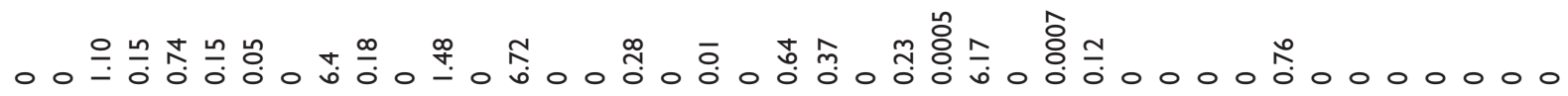

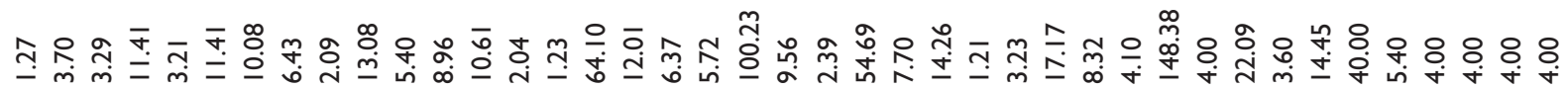

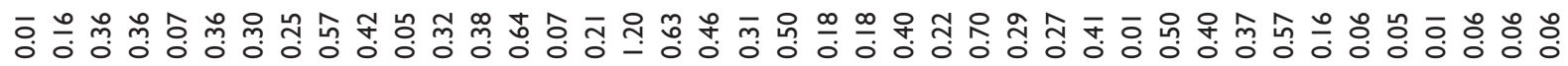

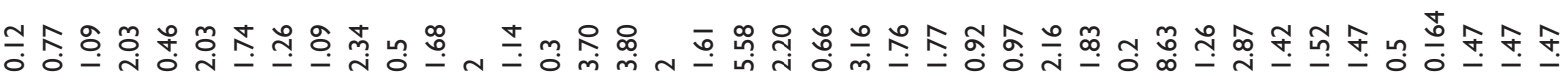

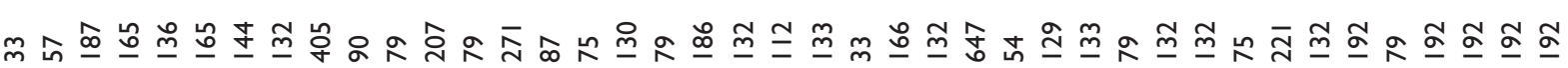

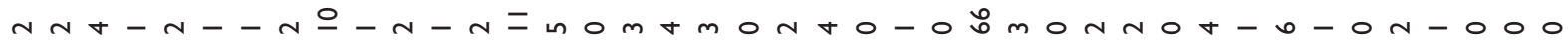



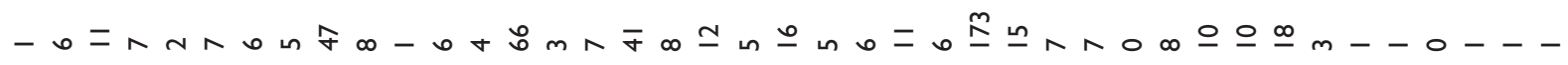

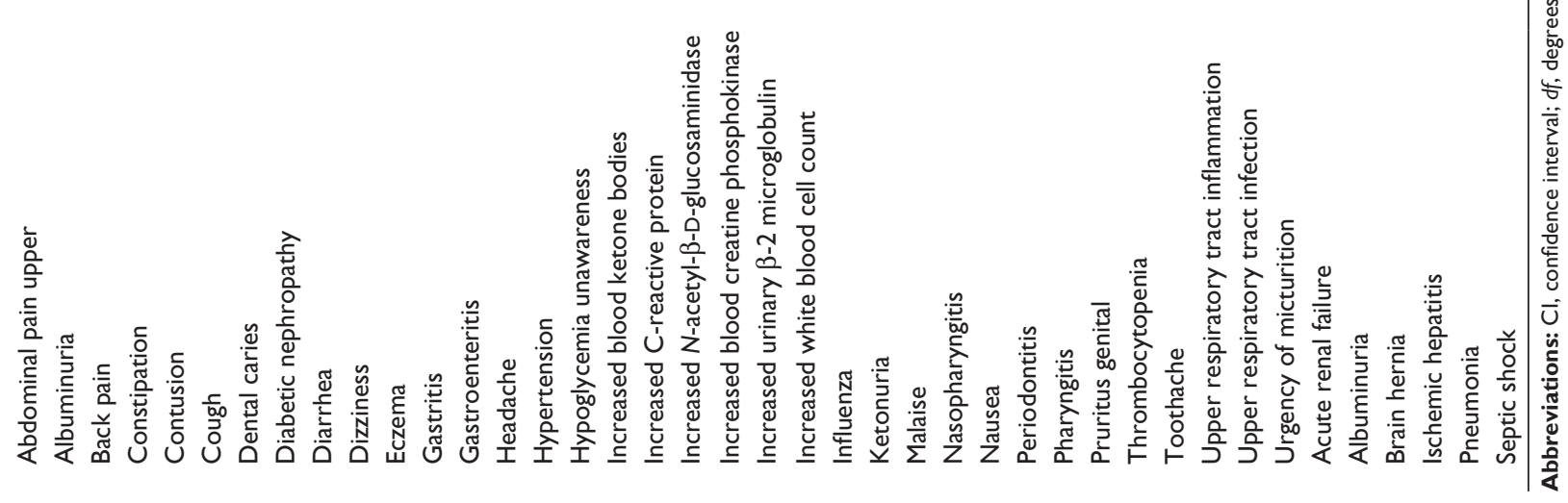




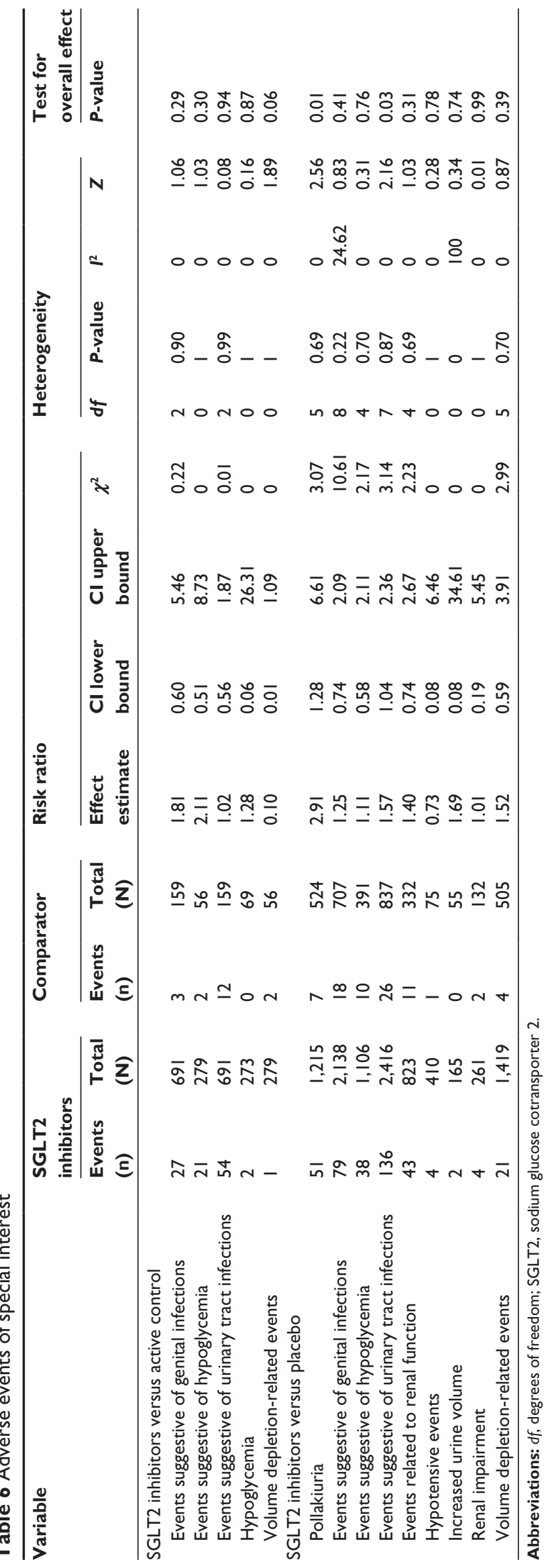

For pollakiuria (six trials; 1,739 participants), patients receiving SGLT2 inhibitors experienced a greater risk compared with patients receiving placebo (relative risk $=2.91$, [95\% CI, 1.28, 6.61], $P=0.01$ ). No other significant difference was found between SGLT2 inhibitor and placebo therapy for any of the other adverse event categories. Similarly, when SGLT2 inhibitors were compared with active control, no significant difference was found between the two treatments for any adverse event category. No heterogeneity was found within trials for either SGLT2 inhibitors versus placebo or SGLT2 inhibitors versus active control $\left(I^{2}=0 \% ; P>0.05\right.$ for all adverse events of special interest).

\section{Discussion}

For this meta-analysis, we selected 13 randomized clinical trials to evaluate the effect of SGLT2 inhibitors monotherapy on glycemic and other clinical laboratory parameters in T2DM patients. In addition, we evaluated the risk of diabetic ketoacidosis and other adverse effects with SGLT2 inhibitors therapy.

\section{$\mathrm{HbAlc}$}

The statistically significant decreases in $\mathrm{HbA} 1 \mathrm{c}$ for patients receiving SGLT2 inhibitors as monotherapy versus those receiving a placebo are similar to results found in other trials in which SGLT inhibitors were used as a background or add-on therapy. In these studies, decreases in HbAlc were observed from baseline to week 26 in the groups receiving $100 \mathrm{mg}$ and $300 \mathrm{mg}$ of canagliflozin with metformin alone or with sulfonylurea, compared with placebo and metformin alone or with sulfonylurea. ${ }^{25-27}$ Similar results were seen in studies of empagliflozin and ipragliflozin in combination with metformin. ${ }^{28,29}$ In these studies, those receiving $10-50 \mathrm{mg}$ doses of empagliflozin and 12.5-300 mg doses of ipragliflozin experienced statistically significant decreases compared with the groups receiving placebo and metformin..$^{28,29}$ In this meta-analysis, no statistically significant changes were found for HbA1c when SGLT2 inhibitors were compared with active control. By contrast, in trials, decreases in HbAlc for SGLT2 inhibitors were comparable with or superior to active control. ${ }^{30,31}$ Such differences could be attributed to the limited number of eligible trials in which SGLT2 inhibitors were compared with active control.

\section{Fasting plasma glucose}

Although notable, decreases in FPG that occurred in the groups receiving SGLT2 inhibitor treatment compared with groups receiving placebo treatment were not statistically 
significant. Similarly, no significant changes for FPG occurred when SGLT2 inhibitors were compared with active control. This is different from other trials in which FPG was significantly decreased among patients receiving SGLT2 inhibitors. In trials in which ipragliflozin was compared with placebo as part of combination therapy, the treatment with ipragliflozin demonstrated statistically significant decreases ( $P \leq 0.05$ for the groups receiving ipragliflozin vs placebo). ${ }^{32}$ Similarly, FPG was significantly decreased in groups receiving empagliflozin as an add-on to metformin in another study. ${ }^{33}$ In other trials in which SGLT2 inhibitors were compared with active controls, SGLT2 inhibitors demonstrated decreases in FPG that were comparable with or were significantly greater than those for active control..$^{30,31}$ A number of factors could account for these discrepancies. For the comparison of SGLT2 inhibitors versus active control, the limited number of eligible trials could have influenced the results. In addition, in some of the trials, SGLT2 inhibitors were used in combination with another antidiabetic treatment. Although the results for SGLT2 inhibitors versus placebo therapy were not statistically significant in this metaanalysis, the $P$-value was 0.07 , indicating that patients receiving SGLT2 inhibitors still experienced notable decreases.

\section{2-hour PPG}

In this meta-analysis, treatment effects that favored SGLT2 inhibitors over placebo therapy were not statistically significant. These results differ from those found in other trials. In a multiple-dose study, 2-hour PPG significantly decreased among patients receiving active doses of canagliflozin. ${ }^{26,27}$ Such differences could possibly be attributed to individual differences among SGLT2 inhibitors. Similarly, in a study in which canagliflozin was compared with dapagliflozin, excursions in PPG decreased and occurred later during canagliflozin treatment versus dapagliflozin treatment. ${ }^{34}$ The authors attributed these delays and decreases in PPG to a temporary and local inhibition of intestinal SGLT1 that is associated with canagliflozin treatment. ${ }^{34}$ This inhibition decreases the rate of absorption of glucose into the intestine. ${ }^{34}$ As such, our inclusion of various SGLT2 inhibitors could have affected the results.

\section{Body weight}

In this meta-analysis, weight significantly decreased for SGLT2 inhibitors versus placebo therapy. Similarly, in trials of SGLT2 inhibitors, modest decreases were found. Trials of dapagliflozin and canagliflozin have revealed that active doses of these treatments resulted in decreased body weight
$\left(P<0.05\right.$ for all doses at all time points). ${ }^{35-37}$ No significant decreases in weight occurred for SGLT2 inhibitors versus active control; this differs from the results seen in other trials. In some trials, SGLT2 inhibitors demonstrated a greater weight reduction than those for active control, ${ }^{30}$ and in one study, these changes were found to be statistically significant. ${ }^{31}$ Results on the effects of other SGLT2 inhibitors on changes in body weight varied. Body weight decreases for two different active doses of dapagliflozin were not statistically significant in some trials. ${ }^{26,28}$ Similarly, in a study of $12.5 \mathrm{mg}, 50 \mathrm{mg}, 150 \mathrm{mg}$, and $300 \mathrm{mg}$ ipragliflozin, the changes that occurred at week 12 were statistically significant only for the three highest doses $(P<0.001$ for all values $) .^{29}$ Possibly contributing to these discrepancies is the use of combination treatments such as metformin, sulfonylureas, glitazones, or insulin. Metformin is known to facilitate weight loss, whereas sulfonylureas, glitazones, and insulin facilitate weight gain. ${ }^{38,39}$ However, weight loss in trials has tended to be modest even when it has been statistically significant. ${ }^{36-39}$

\section{Lipid profile}

Our results regarding the LDL/HDL ratio are similar to those reported in other trials. In this meta-analysis, patients receiving SGLT2 inhibitors experienced statistically significant decreases in LDL/HDL ratios compared with patients receiving placebo therapy. By contrast, small increases in the LDL/HDL ratio were seen among patients receiving canagliflozin. ${ }^{25}$ However, the results regarding triglycerides, HDLs, and LDLs in this meta-analysis are different from those found in other trials. No statistically significant changes were seen for triglycerides, HDL, and LDL in this metaanalysis. By contrast, increases in HDL and LDL occurred among patients receiving canagliflozin and dapagliflozin, whereas triglycerides decreased for patients receiving these agents. ${ }^{25,35,40}$ Such discrepancies could possibly be attributed to the treatments involved. In these trials, SGLT2 inhibitors were administered in combination with another antidiabetic agent such as metformin, sulfonylurea, or pioglitazone, and patients receiving placebos also received one of these agents. ${ }^{25,35,40}$ As such, administration of an additional agent might have affected the results in these trials.

\section{Renal profile}

Renal parameters are of concern for SGLT2 inhibitor therapy because of the effects of SGLT2 inhibition on the kidneys. The inhibition of glucose and sodium resorption leads to changes in renal function. ${ }^{41}$ In this meta-analysis, patients 
receiving SGLT2 inhibitors experienced statistically significant increases in creatinine and moderate decreases in uric acid compared with patients receiving placebo therapy. This differs from results found in other trials. Patients receiving dapagliflozin in trials did not experience significant changes in creatinine, and the changes among patients receiving canagliflozin were small or similar to those for patients receiving placebos. ${ }^{36,42}$ By contrast, the results regarding uric acid are similar to those found in other trials. Patients receiving dapagliflozin experienced significant decreases in uric acid compared with patients receiving placebo therapy. ${ }^{36}$ In this meta-analysis, no significant changes were seen in eGFR; however, the effects of SGLT2 inhibitors on eGFR varied. In studies of ipragliflozin and dapagliflozin administered to patients with renal impairment, eGFR decreased after 1-2 weeks of treatment, stabilized after the first or second week, and either remained stable throughout the course of the study or returned to baseline levels by the end. ${ }^{41,43}$ Similar results were seen in trials of canagliflozin and empagliflozin. ${ }^{26,41}$ By contrast, eGFR increased among patients receiving tofogliflozin. Antihypertensive effects, diuretic effects, and increased tubuloglomerular feedback may influence these initial decreases. ${ }^{41}$

\section{Ketones}

Diabetic ketoacidosis is of concern during treatment with SGLT2 inhibitors because some cases have been reported. ${ }^{44}$ Many occurred during off-label use in patients with type 1 diabetes; however, some cases have occurred in patients with T2DM. ${ }^{44}$ Among patients who participated in randomized controlled trials of canagliflozin, the incidence rates ranged from 0.238 to 0.763 per 1,000 patient-years. ${ }^{45}$ Although other factors, such as blood glucose levels greater than $300 \mathrm{mg} / \mathrm{dL}$, as well as concomitant use of insulin, infections, acute illness, decreased carbohydrate intake, missed insulin doses or pump failures, recent surgical interventions, and alcohol use, could have influenced the development of diabetic ketoacidosis in these patients, such cases have prompted the Food and Drug Administration to issue a warning regarding the condition. ${ }^{41,44,46}$ In this meta-analysis, no statistically significant changes were found for levels of acetoacetic acid, $\beta$-hydroxybutyric acid, and total ketone bodies. However, patients receiving SGLT2 inhibitors were at greater risk of increased ketone bodies compared with patients receiving placebos. These results differ from those found in other trials. Mean levels of ketone bodies, acetoacetic acid, and beta-hydroxybutyric acid increased among patients receiving luseogliflozin and tofogliflozin. ${ }^{45,47-49}$ However, among patients receiving canagliflozin, incidences of diabetic ketoacidosis and associated adverse events were relatively low $(0.03 \%-0.11 \%)$, and the increases in mean total ketone bodies ranged from $131.4 \mu \mathrm{mol} / \mathrm{L}$ to $141.6 \mu \mathrm{mol} / \mathrm{L}$ (reference range $\leq 130 \mu \mathrm{mol} / \mathrm{L}) .{ }^{45,49}$ In this meta-analysis, such discrepancies could be attributed to the limited number of eligible trials in which changes in ketone levels were reported as an efficacy variable.

\section{Safety}

Overall SGLT2 inhibitors were safe and well tolerated. No significant risk was found for discontinuations due to adverse events, serious adverse events, and adverse events related to treatment for patients receiving SGLT2 inhibitors versus those receiving either placebo therapy or active control. No significantly increased overall risk of experiencing an adverse event was found for patients receiving SGLT2 inhibitors versus active control. For patients receiving SGLT2 inhibitors, the overall risk of experiencing an adverse event was only slightly greater than that for patients receiving placebos.

Apart from increased blood ketone bodies, the greatest risk among patients receiving SGLT2 inhibitors compared with either active control or placebo therapy was for events suggestive of urinary tract infections and pollakiuria. In trials, the most frequently reported adverse events among patients receiving treatment with SGLT2 inhibitors are female genital mycotic infections, urinary tract infections, and increased urination. ${ }^{50}$ Thus, the adverse events that occurred within this study were similar to those reported in other trials. Urinary tract-related adverse events are of common concern for patients receiving SGLT2 inhibitors. For events suggestive of urinary tract infection, patients receiving SGLT2 inhibitors experienced a greater risk compared with patients receiving placebo. For pollakiuria, patients receiving SGLT2 inhibitors experienced a greater risk compared with patients receiving placebos. This risk is much greater than the actual incidence rates reported in trials. The rates of urinary tract infections diagnosed among patients receiving dapagliflozin ranged from $3.6 \%$ to $5.7 \%$ and were either similar or slightly higher than those among patients receiving placebos $(3.7 \%) .{ }^{50}$ In a postmarketing surveillance study of ipragliflozin, pollakiuria/ polyuria occurred was $1.32 \% .^{51}$ This suggests that the risk of experiencing these events may be markedly greater than the actual incidences.

Although diarrhea has not been identified as a major adverse event in SGLT2 inhibitor trials, it is still of interest because of the potential of nonselective SGLT inhibitors to cause diarrhea by blocking glucose absorption from the 
intestine. ${ }^{52}$ In this meta-analysis, patients receiving SGLT2 inhibitors did not experience a significantly greater risk of diarrhea than patients receiving placebos, and patients receiving SGLT2 inhibitors were at lower risk of experiencing diarrhea than those receiving an active control. Similarly, the incidences of diarrhea as an adverse event have been low in other trials. In trials in which dapagliflozin was used in combination with metformin, patients receiving dapagliflozin and metformin reported diarrhea at a rate similar to those receiving glipizide and metformin. ${ }^{53}$ Similar results were found for patients receiving dapagliflozin with pioglitazone compared with placebo and pioglitazone. ${ }^{54}$ The safety findings regarding hypoglycemia are similar to those of other trials. In the trials under review, the incidence of hypoglycemia in patients receiving canagliflozin was less than that in those receiving glimepiride. ${ }^{55}$ Similarly, in trials of ipragliflozin and dapagliflozin, they either experienced hypoglycemia at a comparable rate to those receiving placebo therapy or they did not experience it at all. ${ }^{29,54}$

\section{Limitations}

A major limitation of this meta-analysis was the inadequate reporting of the methodology in the included trials. Although only one trial received a "high" score in the selective reporting category of the Cochrane Collaboration Risk of Bias Tool, at least seven trials received an "unclear" score in the categories of random sequence generation, allocation concealment, blinding of participants and personnel, blinding of outcome assessment, and incomplete outcome data. These scores were mostly attributable to discrepancies or incomplete information regarding how randomization was attained, how allocation to a given treatment was concealed from participants and personnel, and how missing data were accounted for. The inadequate reporting of such information could have affected the quality of this meta-analysis.

Another concern is the lack of reporting of the outcomes of interest in the eligible studies, particularly for the assessment of the effects of SGLT2 inhibitors therapy on renal outcomes compared with placebo and active control treatments. The renal parameters such as eGFR, albumin-to-creatinine ratio, and albumin levels for patients receiving SGLT2 inhibitors versus placebo therapy were assessed in one trial, and they were not reported in trials in which SGLT2 inhibitors were compared with active control. Similarly, changes from baseline in BUN and uric acid for SGLT2 inhibitors versus active control were reported in one study. As such, the ability to assess the effect of SGLT2 inhibitors for these variables in comparison with placebo and active control treatments was limited.

Similar issues were found in the assessment of the effect of SGLT2 inhibitors on 2-hour PPG and lipid parameters as compared with active control. Changes in 2-hour PPG, total cholesterol, HDL, LDL, and triglycerides for SGLT2 inhibitors versus active control were not reported in any of the eligible trials. Because none of these variables were assessable, the effect of SGLT2 inhibitor therapy on these variables as compared with other antidiabetic medications could not be determined.

Although inclusion of parameters related to diabetic ketoacidosis was a strength of this meta-analysis, changes in ketone levels were reported in three trials only. As such, the sample size for the assessment of this variable for SGLT2 inhibitors versus placebo therapy was small, which may have skewed the results. Moreover, they were not reported for SGLT2 inhibitors versus active control and as such were not assessable.

As an increase in the glucagon-to-insulin ratio, increased free fatty acids, a shift in substrate oxidation from carbohydrate to fat, and decreases in ketone body clearance may make patients taking SGLT2 inhibitors more susceptible to developing diabetic ketoacidosis, more research is necessary to determine the specific factors responsible for development of this condition. ${ }^{41}$

\section{Conclusion}

Overall, SGLT2 inhibitors significantly decreased HbA1c, body weight, and the LDL/HDL ratio. They were also safe and well tolerated. Compared with either placebo therapy or active control, SGLT2 inhibitors posed no risk for discontinuation due to adverse events, serious adverse events, or adverse events related to treatment. However, given the lack of reporting of renal and lipid parameters, more placebocontrolled trials are necessary to determine their effects on these variables, particularly in comparison with other antidiabetic medications.

\section{Disclosure}

The authors report no conflicts of interest in the work.

\section{References}

1. Shaw JE, Sicree RA, Zimmet PZ. Global estimates of the prevalence of diabetes for 2010 and 2030. Diabetes Res Clin Pract. 2010;87(1):4-14.

2. Whiting DR, Guariguata L, Weil C, Shaw J. IDF diabetes atlas: global estimates of the prevalence of diabetes for 2011 and 2030. Diabetes Res Clin Pract. 2011;94(3):311-321.

3. Jung CH, Jang JE, Park J-Y. A novel therapeutic agent for type 2 diabetes mellitus: SGLT2 Inhibitor. Diabetes Metab J. 2014;38(4):261-273. 
4. DeFronzo RA, Davidson JA, Del Prato S. The role of the kidneys in glucose homeostasis: a new path towards normalizing glycaemia. Diabetes Obes Metab. 2012;14(1):5-14.

5. Gallo LA, Wright EM, Vallon V. Probing SGLT2 as a therapeutic target for diabetes: basic physiology and consequences. Diab Vasc Dis Res. 2015;12(2):78-89.

6. Kim GW, Chung SH. Clinical implication of SGLT2 inhibitors in type 2 diabetes. Arch Pharm Res. 2014;37(8):957-966.

7. Powell J, Miller SA, Taylor JR. Sodium-glucose cotransporter 2 inhibitors: the new option for diabetes mellitus management. South Med J. 2015;108(2):82-90.

8. Markham A, Elkinson S. Luseogliflozin: first global approval. Drugs. 2014;74(8):945-950.

9. Taylor SI, Blau JE, Rother KI. SGLT2 inhibitors may predispose to ketoacidosis. J Clin Endocrinol Metab. 2015;100(8):2849-2852.

10. Higgins JPT, Altman DG, Gøtzsche PC, et al; Cochrane Bias Methods Group; Cochrane Statistical Methods Group. The Cochrane Collaboration's tool for assessing risk of bias in randomised trials. BMJ. 2011; 343:d5928.

11. Barza M, Trikalinos TA, Lau J. Statistical considerations in metaanalysis. Infect Dis Clin North Am. 2009;23(2):195-210.

12. Ferrannini E, Ramos SJ, Salsali A, Tang W, List JF. Dapagliflozin monotherapy in type 2 diabetic patients with inadequate glycemic control by diet and exercise: a randomized, double-blind, placebocontrolled, phase 3 trial. Diabetes Care. 2010;33(10):2217-2224.

13. Ji L, Ma J, Li H, et al. Dapagliflozin as monotherapy in drug-naive Asian patients with type 2 diabetes mellitus: a randomized, blinded, prospective phase III study. Clin Ther. 2014;36(1):84.e-100.e.

14. Kaku K, Kiyosue A, Inoue S, et al. Efficacy and safety of dapagliflozin monotherapy in Japanese patients with type 2 diabetes inadequately controlled by diet and exercise. Diabetes Obes Metab. 2014;16(11): 1102-1110.

15. List JF, Woo V, Morales E, Tang W, Fiedorek FT. Sodium-glucose cotransport inhibition with dapagliflozin in type 2 diabetes. Diabetes Care. 2009;32(4):650-657.

16. Inagaki N, Kondo K, Yoshinari T, Maruyama N, Susuta Y, Kuki H. Efficacy and safety of canagliflozin in Japanese patients with type 2 diabetes: a randomized, double-blind, placebo-controlled, 12-week study. Diabetes Obes Metab. 2013;15(12):1136-1145.

17. Inagaki N, Kondo K, Yoshinari T, Takahashi N, Susuta Y, Kuki H. Efficacy and safety of canagliflozin monotherapy in Japanese patients with type 2 diabetes inadequately controlled with diet and exercise: a 24-week, randomized, double-blind, placebo-controlled, Phase III study. Expert Opin Pharmacother. 2014;15(11):1501-1515.

18. Stenlöf K, Cefalu WT, Kim KA, et al. Efficacy and safety of canagliflozin monotherapy in subjects with type 2 diabetes mellitus inadequately controlled with diet and exercise. Diabetes Obes Metab. 2013; 15(4):372-382.

19. Seino Y, Sasaki T, Fukatsu A, Sakai S, Samukawa Y. Efficacy and safety of luseogliflozin monotherapy in Japanese patients with type 2 diabetes mellitus: a 12-week, randomized, placebo-controlled, phase II study. Curr Med Res Opin. 2014;30(7):1219-1230.

20. Seino Y, Sasaki T, Fukatsu A, Ubukata M, Sakai S, Samukawa Y. Dosefinding study of luseogliflozin in Japanese patients with type 2 diabetes mellitus: a 12-week, randomized, double-blind, placebo-controlled, phase II study. Curr Med Res Opin. 2014;30(7):1231-1244.

21. Seino Y, Sasaki T, Fukatsu A, Ubukata M, Sakai S, Samukawa Y. Efficacy and safety of luseogliflozin as monotherapy in Japanese patients with type 2 diabetes mellitus: a randomized, double-blind, placebo-controlled, phase 3 study. Curr Med Res Opin. 2014;30(7): 1245-1255.

22. Fonseca VA, Ferrannini E, Wilding JP, et al. Active- and placebocontrolled dose-finding study to assess the efficacy, safety, and tolerability of multiple doses of ipragliflozin in patients with type 2 diabetes mellitus. J Diabetes Complications. 2013;27(3):268-273.
23. Kaku K, Watada H, Iwamoto Y, et al; Tofogliflozin 003 Study Group. Efficacy and safety of monotherapy with the novel sodium/glucose cotransporter-2 inhibitor tofogliflozin in Japanese patients with type 2 diabetes mellitus: a combined Phase 2 and 3 randomized, placebocontrolled, double-blind, parallel-group comparative study. Cardiovasc Diabetol. 2014;13:65.

24. Sykes AP, Kemp GL, Dobbins R, et al. Randomized efficacy and safety trial of once-daily remogliflozin etabonate for the treatment of type 2 diabetes. Diabetes Obes Metab. 2015;17(1):98-101.

25. Forst T, Guthrie R, Goldenberg R, et al. Efficacy and safety of canagliflozin over 52 weeks in patients with type 2 diabetes on background metformin and pioglitazone. Diabetes Obes Metab. 2014;16(5): 467-477.

26. Wilding JP, Charpentier G, Hollander P, et al. Efficacy and safety of canagliflozin in patients with type 2 diabetes mellitus inadequately controlled with metformin and sulphonylurea: a randomised trial. Int J Clin Pract. 2013;67(12):1267-1282.

27. Lavalle-González FJ, Januszewicz A, Davidson J, et al. Efficacy and safety of canagliflozin compared with placebo and sitagliptin in patients with type 2 diabetes on background metformin monotherapy: a randomised trial. Diabetologia. 2013;56(12):2582-2592.

28. Rosenstock J, Seman LJ, Jelaska A, et al. Efficacy and safety of empagliflozin, a sodium glucose cotransporter 2 (SGLT2) inhibitor, as add-on to metformin in type 2 diabetes with mild hyperglycaemia. Diabetes Obes Metab. 2013;15(12):1154-1160.

29. Wilding JP, Ferrannini E, Fonseca VA, Wilpshaar W, Dhanjal P, Houzer A. Efficacy and safety of ipragliflozin in patients with type 2 diabetes inadequately controlled on metformin: a dose-finding study. Diabetes Obes Metab. 2013;15(5):403-409.

30. Ferrannini E, Berk A, Hantel S, et al. Long-term safety and efficacy of empagliflozin, sitagliptin, and metformin: an active-controlled, parallelgroup, randomized, 78-week open-label extension study in patients with type 2 diabetes. Diabetes Care. 2013;36(12):4015-4021.

31. Schernthaner G, Gross JL, Rosenstock J, et al. Canagliflozin compared with sitagliptin for patients with type 2 diabetes who do not have adequate glycemic control with metformin plus sulfonylurea: a 52-week randomized trial. Diabetes Care. 2013;36(9):2508-2515.

32. Kashiwagi A, Kazuta K, Goto K, Yoshida S, Ueyama E, Utsuno A. Ipragliflozin in combination with metformin for the treatment of Japanese patients with type 2 diabetes: ILLUMINATE, a randomized, double-blind, placebo-controlled study. Diabetes Obes Metab. 2015; 17(3):304-308.

33. Häring HU, Merker L, Seewaldt-Becker E, et al; EMPA-REG MET Trial Investigators. Empagliflozin as add-on to metformin in patients with type 2 diabetes: a 24-week, randomized, double-blind, placebocontrolled trial. Diabetes Care. 2014;37(6):1650-1659.

34. Sha S, Polidori D, Farrell K, et al. Pharmacodynamic differences between canagliflozin and dapagliflozin: results of a randomized, double-blind, crossover study. Diabetes Obes Metab. 2015;17(2):188-197.

35. Ji L, Han P, Liu Y, et al. Canagliflozin in Asian patients with type 2 diabetes on metformin alone or metformin in combination with sulphonylurea. Diabetes Obes Metab. 2015;17(1):23-31.

36. Bailey CJ, Gross JL, Hennicken D, Iqbal N, Mansfield TA, List JF. Dapagliflozin add-on to metformin in type 2 diabetes inadequately controlled with metformin: a randomized, double-blind, placebocontrolled 102-week trial. BMC Med. 2013;11:43.

37. Bolinder J, Ljunggren Ö, Kullberg J, et al. Effects of dapagliflozin on body weight, total fat mass, and regional adipose tissue distribution in patients with type 2 diabetes mellitus with inadequate glycemic control on metformin. J Clin Endocrinol Metab. 2012;97(3):1020-1031.

38. Rojas LBA, Gomes MB. Metformin: an old but still the best treatment for type 2 diabetes. Diabetol Metab Syndr. 2013;5(1):6.

39. Clar C, Gill JA, Court R, Waugh N. Systematic review of SGLT2 receptor inhibitors in dual or triple therapy in type 2 diabetes. BMJ Open. 2012;2(5):e001007. 
40. Matthaei S, Bowering K, Rohwedder K, et al. Durability and tolerability of dapagliflozin over 52 weeks as add-on to metformin and sulphonylurea in type 2 diabetes. Diabetes Obes Metab. 2015;17(11):1075-1084.

41. Mudaliar S, Polidori D, Zambrowicz B, Henry RR. Sodium-glucose cotransporter inhibitors: effects on renal and intestinal glucose transport: from bench to bedside. Diabetes Care. 2015;38(12):2344-2353.

42. Strojek K, Yoon KH, Hruba V, et al. Effect of dapagliflozin in patients with type 2 diabetes who have inadequate glycaemic control with glimepiride: a randomized, 24-week, double-blind, placebo-controlled trial. Diabetes Obes Metab. 2011;13(10):928-938.

43. Kashiwagi A, Takahashi $\mathrm{H}$, Ishikawa $\mathrm{H}$, et al. A randomized, doubleblind, placebo-controlled study on long-term efficacy and safety of ipragliflozin treatment in patients with type 2 diabetes mellitus and renal impairment: results of the long-term ASP1941 safety evaluation in patients with type 2 diabetes with renal impairment (LANTERN) study. Diabetes Obes Metab. 2015;17(2):152-160.

44. Peters AL, Buschur EO, Buse JB, Cohan P, Diner JC, Hirsch IB. Euglycemic diabetic ketoacidosis: a potential complication of treatment with sodium-glucose cotransporter 2 inhibition. Diabetes Care. 2015 38(9):1687-1693.

45. Erondu N, Desai M, Ways K, Meininger G. Diabetic ketoacidosis and related events in the canagliflozin type 2 diabetes clinical program. Diabetes Care. 2015;38(9):1680-1686.

46. Food and Drug Administration [webpage on the Internet]. FDA Drug Safety Communication: FDA warns that SGLT2 inhibitors for diabetes may result in a serious condition of too much acid in the blood; 2015. Available from: http://www.fda.gov/Drugs/DrugSafety/ucm446845. htm. Accessed March 30, 2016.

47. Tanizawa Y, Kaku K, Araki E, et al; Tofogliflozin 004 and 005 Study Group. Long-term safety and efficacy of tofogliflozin, a selective inhibitor of sodium-glucose cotransporter 2, as monotherapy or in combination with other oral antidiabetic agents in Japanese patients with type 2 diabetes mellitus: multicenter, open-label, randomized controlled trials. Expert Opin Pharmacother. 2014;15(6):749-766.
48. Seino Y, Kaku K, Inagaki N, et al. Fifty-two-week long-term clinical study of luseogliflozin as monotherapy in Japanese patients with type 2 diabetes mellitus inadequately controlled with diet and exercise. Endocr J. 2015;62(7):593-603.

49. Inagaki N, Kondo K, Yoshinari T, Kuki H. Efficacy and safety of canagliflozin alone or as add-on to other oral antihyperglycemic drugs in Japanese patients with type 2 diabetes: a 52-week open-label study. $J$ Diabetes Investig. 2015;6(2):210-218.

50. Halimi S, Vergès B. Adverse effects and safety of SGLT-2 inhibitors. Diabetes Metab. 2014;40(6 Suppl 1):S28-S34.

51. Terauchi Y, Yokote K, Nakamura I, Sugamori H. Safety of ipragliflozin in elderly Japanese patients with type 2 diabetes mellitus (STELLAELDER): interim results of a post-marketing surveillance study. Expert Opin Pharmacother. 2016;17(4):463-471.

52. Kurosaki E, Ogasawara H. Ipragliflozin and other sodium-glucose cotransporter-2 (SGLT2) inhibitors in the treatment of type 2 diabetes: preclinical and clinical data. Pharmacol Ther. 2013;139(1):51-59.

53. Nauck MA, Del Prato S, Meier JJ, et al. Dapagliflozin versus glipizide as add-on therapy in patients with type 2 diabetes who have inadequate glycemic control with metformin: a randomized, 52-week, doubleblind, active-controlled noninferiority trial. Diabetes Care. 2011;34(9): 2015-2022.

54. Rosenstock J, Vico M, Wei L, Salsali A, List JF. Effects of dapagliflozin, an SGLT2 inhibitor, on $\mathrm{HbA}(1 \mathrm{c})$, body weight, and hypoglycemia risk in patients with type 2 diabetes inadequately controlled on pioglitazone monotherapy. Diabetes Care. 2012;35(7):1473-1478.

55. Leiter LA, Yoon KH, Arias P, et al. Canagliflozin provides durable glycemic improvements and body weight reduction over 104 weeks versus glimepiride in patients with type 2 diabetes on metformin: a randomized, double-blind, phase 3 study. Diabetes Care. 2015;38(3):355-364.
Therapeutics and Clinical Risk Management

\section{Publish your work in this journal}

Therapeutics and Clinical Risk Management is an international, peerreviewed journal of clinical therapeutics and risk management, focusing on concise rapid reporting of clinical studies in all therapeutic areas, outcomes, safety, and programs for the effective, safe, and sustained use of medicines. This journal is indexed on PubMed Central, CAS,

\section{Dovepress}

EMBase, Scopus and the Elsevier Bibliographic databases. The manuscript management system is completely online and includes a very quick and fair peer-review system, which is all easy to use. Visit http://www.dovepress.com/testimonials.php to read real quotes from published authors. 